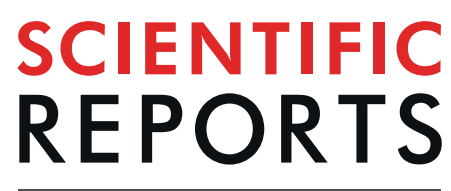

natureresearch

Check for updates

\title{
Irritable bowel syndrome increases the risk of chronic obstructive pulmonary disease: A retrospective cohort study
}

\author{
Hsiang-Chun Lai ${ }^{1}$, Hung-Jen Lin ${ }^{1,2}$, Yi-Wei Kao ${ }^{3,4}$, Kai-Hsun Wang ${ }^{3,4}$, Jen-Wei Chou ${ }^{5}$, \\ Ben-Chang Shia ${ }^{4,6} \bowtie$ \& Sheng-Teng Huang ${ }^{1,2,7,8,9,10 凶}$
}

Both inflammation and infection are associated with the development of irritable bowel syndrome (IBS) and chronic obstructive pulmonary disease (COPD). The purpose of this study is to further elucidate the association between IBS and COPD through a retrospective cohort study. We enrolled IBS patients diagnosed between 2000 and 2011 with follow-up for at least one year. The non-IBS patients as comparison group were selected with 1:3 matching by propensity score. Statistical analysis was utilized to assess the differences in characteristic distribution, and to compare the cumulative incidence of COPD between the IBS and non-IBS cohorts. We selected 14,021 IBS patients and 42,068 non-IBS patients for comparison. The IBS patients exhibited a significant risk to develop COPD compared with non-IBS patients. Additionally, the cumulative incidence rate of COPD in the IBS cohort increased significantly during the follow-up period of more than ten years, compared to the non-IBS cohort, based on the Kaplan-Meier analysis. The risk of COPD was also significantly decreased in those patients with more than eighteen IBS-related clinical visits. This retrospective cohort study demonstrates the significantly increased risk of COPD in patients with IBS. Therefore, early inspection and prevention of COPD is essential for patients with IBS.

Irritable bowel syndrome (IBS) is a common functional gastrointestinal (GI) disease, more commonly affecting women than men. The global prevalence of IBS reported is 11\%; with the highest reported rate in South America (21.0\%), and the lowest in Southeast Asia $(7.0 \%)^{1}$. Based on the newest Rome IV system, IBS was diagnosed as recurrent abdominal pain at least 1 day/week on average during the prior 3-month period, with patients exhibiting symptoms including a change of abdominal pain pattern after defecation, and a change in the frequency of stool or the form of stool, based on the Bristol stool form scale ${ }^{2}$. These symptoms were generally believed to be related to IBS alone, with no association with any other organic diseases. Meanwhile, Paudel et al. has reported that $27 \%$ of IBS patients were associated with organic lesions, according to the Rome IV criteria, the most prevalent being nonspecific colitis, ileal erosions, polyps, hemorrhoids, and diverticula ${ }^{3}$. The pathophysiology of IBS includes intestinal permeability, gut immune dysfunction, gut bacteria flora overgrowth/alteration, gut motility, visceral sensation, brain-gut axis dysregulation, and changes to psychological status ${ }^{4}$. Comorbidities of IBS are often related to GI disorders, including functional dyspepsia and gastroesophageal reflux disease (GERD); however, comorbidities may be related to extra-GI disorders, such as asthma, fibromyalgia, chronic pelvic pain, interstitial cystitis, migraine, chronic fatigue syndrome, depression, and anxiety ${ }^{5-8}$.

\footnotetext{
${ }^{1}$ Department of Chinese Medicine, China Medical University Hospital, Taichung, Taiwan. ${ }^{2}$ School of Chinese Medicine, China Medical University, Taichung, Taiwan. ${ }^{3}$ Graduate Institute of Business Administration, Fu Jen Catholic University, Taipei, Taiwan. ${ }^{4}$ Research Center of Big Data, College of management, Taipei Medical University, Taipei, Taiwan. ${ }^{5}$ Division of Gastroenterology and Hepatology, Department of Internal Medicine, China Medical University Hospital, Taichung, Taiwan. ${ }^{6}$ Executive Master Program of Business Administration in Biotechnology, College of management, Taipei Medical University, Taipei, Taiwan. ${ }^{7}$ Cancer Research Center for Traditional Chinese Medicine, Department of Medical Research, China Medical University Hospital, Taichung, Taiwan. ${ }^{8}$ Chinese Medicine Research Center, China Medical University, Taichung, Taiwan. ${ }^{9}$ Research Center for Chinese Herbal Medicine, China Medical University, Taichung, Taiwan. ${ }^{10} \mathrm{An}-\mathrm{Nan}$ Hospital, China Medical University, Tainan, Taiwan. ${ }^{凶}$ e-mail: stat1001@tmu. edu.tw; sheng.teng@yahoo.com
} 


\begin{tabular}{|c|c|c|c|c|c|}
\hline \multirow[b]{3}{*}{ Variables } & \multicolumn{4}{|l|}{ IBS } & \multirow[b]{3}{*}{$p$-value } \\
\hline & \multicolumn{2}{|c|}{ Yes $(\mathrm{N}=14,021)$} & \multicolumn{2}{|c|}{ No $(\mathrm{N}=42,068)$} & \\
\hline & $\mathbf{n}$ & $\%$ & $\mathbf{n}$ & $\%$ & \\
\hline Sex & & & & & 0.911 \\
\hline Male & 6354 & 45.3 & 19089 & 45.4 & \\
\hline Female & 7667 & 54.7 & 22979 & 54.6 & \\
\hline Area & & & & & 0.987 \\
\hline Northern & 6506 & 46.4 & 19551 & 46.5 & \\
\hline Central & 3512 & 25.0 & 10569 & 25.1 & \\
\hline Southern & 3748 & 26.7 & 11194 & 26.6 & \\
\hline Eastern & 255 & 1.8 & 754 & 1.8 & \\
\hline Salary & & & & & 0.597 \\
\hline$\leq \mathrm{NT} \$ 15,840$ & 4956 & 35.3 & 14936 & 35.5 & \\
\hline NT\$15,841-25,000 & 5456 & 38.9 & 16484 & 39.2 & \\
\hline$\geq \mathrm{NT} \$ 25,001$ & 3609 & 25.7 & 10648 & 25.3 & \\
\hline Age (years) & & & & & 0.799 \\
\hline $18 \sim 29$ & 1660 & 11.8 & 5023 & 11.9 & \\
\hline $30 \sim 39$ & 2292 & 16.3 & 6784 & 16.1 & \\
\hline $40 \sim 49$ & 3154 & 22.5 & 9349 & 22.2 & \\
\hline $50 \sim 59$ & 2913 & 20.8 & 8648 & 20.6 & \\
\hline $60 \sim 69$ & 2045 & 14.6 & 6263 & 14.9 & \\
\hline$>69$ & 1957 & 14.0 & 6001 & 14.3 & \\
\hline Smoking-related diseases/conditions & & & & & 0.056 \\
\hline Yes & 215 & 1.5 & 552 & 1.3 & \\
\hline No & 13806 & 98.5 & 41516 & 98.7 & \\
\hline \multicolumn{6}{|l|}{ Comorbidity } \\
\hline hypertension & 3167 & 22.6 & 10085 & 24 & 0.001 \\
\hline coronary heart disease & 986 & 7.0 & 2949 & 7.0 & 0.944 \\
\hline ischemic stroke & 139 & 1.0 & 407 & 1.0 & 0.842 \\
\hline hemorrhagic stroke & 45 & 0.3 & 177 & 0.4 & 0.121 \\
\hline diabetes mellitus & 1481 & 10.6 & 4588 & 10.9 & 0.264 \\
\hline renal insufficiency & 194 & 1.4 & 577 & 1.4 & 0.949 \\
\hline depression & 417 & 3.0 & 1177 & 2.8 & 0.290 \\
\hline asthma & 329 & 2.3 & 1046 & 2.5 & 0.370 \\
\hline GERD & 174 & 1.2 & 310 & 0.7 & $<0.001$ \\
\hline anxiety & 947 & 6.8 & 2870 & 6.8 & 0.796 \\
\hline osteoporosis & 485 & 3.5 & 1361 & 3.2 & 0.208 \\
\hline peptic ulcer & 720 & 5.1 & 2260 & 5.4 & 0.288 \\
\hline \multicolumn{6}{|l|}{ Medication } \\
\hline steroids & 180 & 1.3 & 538 & 1.3 & $>0.999$ \\
\hline anti-cholinergics & 27 & 0.2 & 70 & 0.1 & 0.600 \\
\hline beta-2 agonist & 155 & 1.1 & 487 & 1.2 & 0.650 \\
\hline
\end{tabular}

Table 1. Demographic Characteristics and comorbidities of patients newly diagnosed with IBS and non-IBS in Taiwan. IBS = irritable bowel syndrome, COPD = chronic obstructive pulmonary disease, $\mathrm{GERD}=$ gastroesophageal reflux disease; Chi-square test was used to test categorical variables.

Chronic obstructive pulmonary disease (COPD) is an obstructive lung disease characterized by shortness of breath and productive cough ${ }^{9}$. The global prevalence rate of COPD is reported to range from $7 \%$ to $12 \%$, affecting males more than females, and resulting in significant social and economic burden ${ }^{10}$. Comorbidities of COPD include pulmonary hypertension, hypertension, congestive heart failure, coronary heart disease, metabolic syndrome, obstructive sleep apnea syndrome, asthma, and GERD ${ }^{11,12}$. Several factors have been reported to trigger COPD, including smoking, genetic determinants, cancer growth, infection, environmental stimuli, and progressive immunological disorders ${ }^{13}$.

Both inflammation and infection models have been utilized in the explorations of IBS and COPD pathophysiology, including investigations into the changes to microbiota, which may play an important role in IBS patients ${ }^{14}$. Although several studies have suggested a higher prevalence of GI disorders among COPD patients, especially GERD ${ }^{15-17}$, less research has explored the correlation between IBS and COPD ${ }^{18}$. We propose that there exists an association between IBS and COPD due to their similar pathophysiological pathways. Herein is a population-based retrospective cohort study of the Taiwan National Health Insurance Research Database (NHIRD) to investigate the association between IBS and COPD. 


\begin{tabular}{|c|c|c|c|c|}
\hline \multirow[b]{2}{*}{ Variables } & \multicolumn{2}{|l|}{ Univariate } & \multicolumn{2}{|l|}{ Multivariate } \\
\hline & Crude HR $(95 \% \mathrm{CI})$ & $P$-value & $\operatorname{aHR}(95 \% \mathrm{CI})^{\dagger}$ & $P$-value \\
\hline \multicolumn{5}{|l|}{ Group } \\
\hline Non-IBS & 1 & reference & 1 & reference \\
\hline IBS & $1.462(1.367,1.564)$ & $<0.0001$ & $1.512(1.414,1.617)$ & $<0.0001$ \\
\hline \multicolumn{5}{|l|}{ Sex } \\
\hline Female & 1 & reference & 1 & reference \\
\hline Male & $1.480(1.389,1.577)$ & $<0.0001$ & $1.610(1.508,1.720)$ & $<0.0001$ \\
\hline \multicolumn{5}{|l|}{ Age } \\
\hline $18 \sim 29$ & 1 & reference & 1 & reference \\
\hline $30 \sim 39$ & $1.652(1.281,2.131)$ & 0.0001 & $1.629(1.261,2.105)$ & 0.0002 \\
\hline $40 \sim 49$ & $2.852(2.265,3.591)$ & $<0.0001$ & $2.777(2.200,3.505)$ & $<0.0001$ \\
\hline $50 \sim 59$ & $5.405(4.322,6.759)$ & $<0.0001$ & $5.097(4.062,6.394)$ & $<0.0001$ \\
\hline $60 \sim 69$ & $9.480(7.601,11.824)$ & $<0.0001$ & $8.395(6.704,10.513)$ & $<0.0001$ \\
\hline$>69$ & $18.241(14.675,22.673)$ & $<0.0001$ & $15.022(12.019,18.776)$ & $<0.0001$ \\
\hline \multicolumn{5}{|l|}{ Area } \\
\hline Northern & 1 & reference & 1 & reference \\
\hline Central & $1.123(1.038,1.215)$ & 0.0038 & $1.020(0.941,1.107)$ & 0.6275 \\
\hline Southern & $1.160(1.075,1.251)$ & 0.0001 & $1.024(0.947,1.106)$ & 0.5551 \\
\hline Eastern & $1.545(1.261,1.892)$ & $<0.0001$ & $1.243(1.012,1.526)$ & 0.0377 \\
\hline \multicolumn{5}{|l|}{ salary } \\
\hline$\leq \mathrm{NT} \$ 15,840$ & 1 & reference & 1 & reference \\
\hline NT\$15,841-25,000 & $0.882(0.824,0.944)$ & 0.0003 & $1.056(0.982,1.136)$ & 0.1404 \\
\hline$\geq \mathrm{NT} \$ 25,001$ & $0.487(0.442,0.536)$ & $<0.0001$ & $0.834(0.749,0.928)$ & 0.0008 \\
\hline \multicolumn{5}{|c|}{ Smoking-related diseases/conditions } \\
\hline No & 1 & reference & 1 & reference \\
\hline Yes & $1.695(1.292,2.224)$ & 0.0001 & $1.190(0.905,1.546)$ & 0.2123 \\
\hline \multicolumn{5}{|l|}{ Comorbidities } \\
\hline hypertension & $2.431(2.279,2.593)$ & $<0.0001$ & $1.057(0.982,1.138)$ & 0.1366 \\
\hline coronary heart disease & $2.532(2.314,2.771)$ & $<0.0001$ & $1.191(1.082,1.310)$ & 0.0003 \\
\hline ischemic stroke & $2.996(2.372,3.784)$ & $<0.0001$ & $1.253(0.989,1.589)$ & 0.0615 \\
\hline hemorrhagic stroke & $2.673(1.916,3.728)$ & $<0.0001$ & $1.396(0.998,1.952)$ & 0.0516 \\
\hline diabetes mellitus & $1.894(1.740,2.062)$ & $<0.0001$ & $1.112(1.016,1.216)$ & 0.0213 \\
\hline renal insufficiency & $2.219(1.810,2.720)$ & $<0.0001$ & $1.131(0.921,1.389)$ & 0.2385 \\
\hline depression & $1.441(1.220,1.702)$ & $<0.0001$ & $1.352(1.143,1.598)$ & 0.0004 \\
\hline asthma & $2.788(2.438,3.188)$ & $<0.0001$ & $2.491(2.177,2.850)$ & $<0.0001$ \\
\hline GERD & $1.570(1.067,2.310)$ & 0.0221 & $1.074(0.729,1.582)$ & 0.7172 \\
\hline anxiety & $1.616(1.453,1.796)$ & $<0.0001$ & $1.155(1.036,1.287)$ & 0.0093 \\
\hline sleep apnea & $1.844(1.047,3.251)$ & 0.0342 & $1.775(1.006,3.135)$ & 0.0478 \\
\hline osteoporosis & $2.025(1.783,2.300)$ & $<0.0001$ & $1.234(1.082,1.407)$ & 0.0017 \\
\hline peptic ulcer & $1.676(1.493,1.881)$ & $<0.0001$ & $1.164(1.034,1.310)$ & 0.0122 \\
\hline
\end{tabular}

Table 2. Cox model with hazard ratios and 95\% confidence intervals of risk associated with COPD and comorbidities in patients with IBS compared to those without IBS. Crude HR= relative hazard ratio, $\mathrm{aHR}=$ Adjusted hazard ratio, $\mathrm{CI}=$ confidence interval; $\mathrm{IBS}=$ irritable bowel syndrome, $\mathrm{COPD}=$ chronic obstructive pulmonary disease, GERD = gastroesophageal reflux disease. ${ }^{\dagger}$ Model was adjusted for age, sex, and comorbidities.

\section{Results}

Demographic characteristics. There were a total of 14,021 subjects included in the IBS cohort, and 42,068 subjects in the non-IBS cohort, as shown in Table 1. The data distribution between the two cohorts was basically similar in terms of sex, residential area, salary, age distribution, smoking-related diseases/conditions, comorbidities, and drug use. Females exhibited a higher incidence of IBS in comparison with men (men: women $=45.3$ : 54.7). People living in the northern region of Taiwan had a higher percentage (46.4\%) of IBS compared to inhabitants of the other regions. It is noteworthy that those patients with higher stated income had lower incidence of IBS (25.7\%). The IBS patients aged from 40-59 had the highest incidence (43.3\%) compared to those less than 40 years of age, and older than 60 years of age $(28.1 \%, 28.6 \%$ respectively). The subjects visited the tobacco quit clinic in both IBS and non-IBS cohorts were $215(1.5 \%)$ and $552(1.3 \%)$ respectively with no statistical significance. In the comparison of comorbidities between the two cohorts, no statistical significance was noted, except for hypertension ( $\mathrm{p}$ value $=0.001$ ), and GERD ( $\mathrm{p}$ value $<0.0001$ ). In addition, no statistical significance was noted in COPD medications between the IBS and non-IBS cohorts. 


\begin{tabular}{|c|c|c|c|c|c|c|c|c|}
\hline \multirow[b]{3}{*}{ Variables } & \multicolumn{6}{|c|}{ Irritable bowel syndrome } & \multirow[b]{3}{*}{ Crude HR ( $95 \%$ CI) } & \multirow[b]{3}{*}{ Adjusted $\mathrm{HR}^{\dagger}(\mathbf{9 5} \% \mathrm{CI})$} \\
\hline & \multicolumn{3}{|c|}{ No } & \multicolumn{3}{|l|}{ Yes } & & \\
\hline & Event & PY & $\mathbf{I R}^{\#}$ & Event & PY & $\mathbf{I R}^{*}$ & & \\
\hline \multicolumn{9}{|l|}{ Age, years } \\
\hline$<=49$ & 449 & 130574.55 & 3.44 & 283 & 44288.76 & 6.39 & $1.85(1.60,2.15)^{* * * *}$ & $1.87(1.61,2.17)^{* * *}$ \\
\hline $50-64$ & 737 & 68117.56 & 10.82 & 407 & 22531.11 & 18.06 & $1.67(1.48,1.89)^{* * *}$ & $1.68(1.49,1.90)^{* * *}$ \\
\hline $65+$ & 1408 & 48534.21 & 29.01 & 573 & 15570.33 & 36.80 & $1.27(1.16,1.40)^{* * * *}$ & $1.31(1.18,1.44)^{* * *}$ \\
\hline \multicolumn{9}{|l|}{ Sex } \\
\hline Female & 1173 & 135804.23 & 8.64 & 575 & 45573.91 & 12.62 & $1.46(1.32,1.61)^{* * *}$ & $1.50(1.35,1.65)^{* * *}$ \\
\hline Male & 1421 & 111422.09 & 12.75 & 688 & 36816.29 & 18.69 & $1.47(1.34,1.61)^{* * *}$ & $1.55(1.41,1.69)^{* * *}$ \\
\hline \multicolumn{9}{|c|}{ Comorbidity $^{\S}$} \\
\hline No & 849 & 143727.02 & 5.91 & 475 & 47446.79 & 10.01 & $1.69(1.51,1.90)^{* * *}$ & $1.76(1.57,1.97)^{* * *}$ \\
\hline Yes & 1745 & 103499.29 & 16.86 & 788 & 34943.41 & 22.55 & $1.34(1.23,1.46)^{* * *}$ & $1.39(1.28,1.51)^{* * *}$ \\
\hline
\end{tabular}

Table 3. Incidence of COPD by age, sex and comorbidity and Cox model measured hazards ratio for patients with IBS compared those without IBS. Crude $\mathrm{HR}=$ relative hazard ratio, aHR = Adjusted hazard ratio, $\mathrm{CI}=$ confidence interval; $\mathrm{IBS}=$ irritable bowel syndrome, $\mathrm{COPD}=$ chronic obstructive pulmonary disease, $\mathrm{IR}=$ incidence rate, $\mathrm{PY}=$ person-years, ${ }^{\dagger}$ Model was adjusted for age, sex, and comorbidities \# incidence rate, per 1000 person-years $\$$ Individuals with any comorbidity of hypertension, coronary heart disease, ischemic stroke, hemorrhagic stroke, diabetes mellitus, renal insufficiency, depression, asthma, gastroesophageal reflux disease, anxiety, sleep apnea, osteoporosis and peptic ulcer were classified into the comorbidity group. $* * * \mathrm{p}<0.001$.

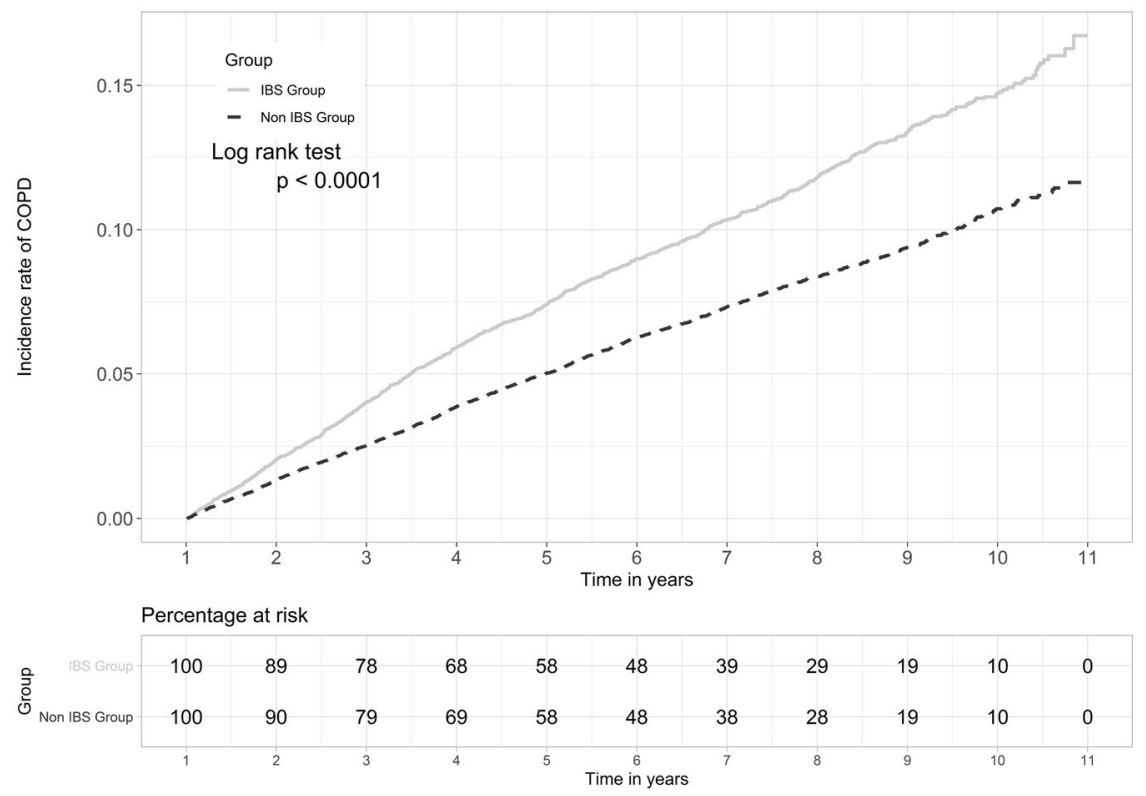

Figure 1. Cumulative incidence of COPD in the patients with IBS and non-IBS by Kaplan-Meier method analysis. The statistical analyses were performed using R software Version 3.4.3 (http://www.r-project.org), with level $\alpha=0.05$ to indicate statistical significance.

The cause-specific hazard ratio and cumulative incidence of COPD. According to the Cox proportional hazards model, the factors affecting COPD risk between the IBS and non-IBS patients included gender, age, salary, residential area, smoking-related diseases/conditions, comorbidities, and COPD medications (Table 2). The results showed that the IBS cohort presented an increased risk of developing COPD compared to the non-IBS cohort (adjusted $\mathrm{HR}=1.512,95 \% \mathrm{CI}=1.414-1.617$, $\mathrm{p}$ value $<0.0001$ ). Of note, during the study period, the cumulative incidence of COPD was significantly higher among IBS patients as compared with non-IBS patients (Fig. 1). In addition, an increased risk of COPD incidence in both cohorts was noted in the male population $(\mathrm{aHR}=1.610,95 \% \mathrm{CI}=1.508-1.720$, $\mathrm{p}$ value $<0.0001)$, and the eastern region inhabitants $(\mathrm{aHR}=1.243,95 \%$ $\mathrm{CI}=1.012-1.526$, $\mathrm{p}$ value $=0.0377)$; whereas a decreased risk of COPD incidence was noted in the population with higher reported income $(\mathrm{aHR}=0.834,95 \% \mathrm{CI}=0.749-0.928$, $\mathrm{p}$ value $=0.0008)$. Meanwhile, age seems to be an important factor, with an increased risk of developing COPD trending parallel with increased age. Positive smoking-related diseases/conditions posed a higher risk of COPD $(\mathrm{HR}=1.695,95 \% \mathrm{CI}=1.292-2.224$, $\mathrm{p}$ value $=0.0001)$, while didn't reach a statistical significance in adjusted $\mathrm{HR}(\mathrm{aHR}=1.190,95 \% \mathrm{CI}=0.905-1.546, \mathrm{p}$ 


\begin{tabular}{|c|c|c|c|c|}
\hline \multirow[b]{2}{*}{ Medication (days) } & \multicolumn{2}{|l|}{ Univariate } & \multicolumn{2}{|l|}{ Multivariate } \\
\hline & Crude HR $(95 \% \mathrm{CI})$ & $P$-value & $\operatorname{aHR}(95 \% \mathrm{CI})^{\dagger}$ & $P$-value \\
\hline \multicolumn{5}{|l|}{ Steroids } \\
\hline \multicolumn{5}{|l|}{ less than 28 days } \\
\hline non IBS & 1 & reference & 1 & reference \\
\hline IBS & $1.461(1.365,1.563)$ & $<0.0001$ & $1.514(1.415,1.620)$ & $<0.0001$ \\
\hline \multicolumn{5}{|l|}{28 to 84 days } \\
\hline non IBS & 1 & reference & 1 & reference \\
\hline IBS & $2.653(1.117,6.302)$ & 0.0271 & $2.337(0.926,5.894)$ & 0.0722 \\
\hline \multicolumn{5}{|l|}{ more than 84 days } \\
\hline non IBS & 1 & reference & 1 & reference \\
\hline I BS & $0.318(0.040,2.545)$ & 0.2804 & $0.122(0.008,1.912)$ & 0.1339 \\
\hline \multicolumn{5}{|l|}{ Anti-cholinergics } \\
\hline \multicolumn{5}{|l|}{ less than 28 days } \\
\hline non IBS & 1 & reference & 1 & reference \\
\hline IBS & $1.464(1.368,1.566)$ & $<0.0001$ & $1.514(1.415,1.62)$ & $<0.0001$ \\
\hline \multicolumn{5}{|l|}{28 to 84 days } \\
\hline non IBS & 1 & reference & 1 & reference \\
\hline IBS & $0.580(0.171,1.97)$ & 0.3825 & $0.181(0.020,1.677)$ & 0.1325 \\
\hline \multicolumn{5}{|l|}{ more than 84 days } \\
\hline non IBS & 1 & reference & 1 & reference \\
\hline IBS & $1.412(0.56,3.563)$ & 0.4647 & $0.028(0.001,0.680)$ & 0.0281 \\
\hline \multicolumn{5}{|l|}{$\beta 2$ agonist } \\
\hline \multicolumn{5}{|l|}{ less than 28 days } \\
\hline non IBS & 1 & reference & 1 & reference \\
\hline IBS & $1.459(1.363,1.562)$ & $<0.0001$ & $1.514(1.414,1.621)$ & $<0.0001$ \\
\hline \multicolumn{5}{|l|}{28 to 84 days } \\
\hline non IBS & 1 & reference & 1 & reference \\
\hline IBS & $1.569(0.927,2.656)$ & \begin{tabular}{|l|}
0.0934 \\
\end{tabular} & $1.170(0.632,2.165)$ & 0.6175 \\
\hline \multicolumn{5}{|l|}{ more than 84 days } \\
\hline non IBS & 1 & reference & 1 & reference \\
\hline IBS & $1.858(0.926,3.729)$ & 0.0815 & $1.432(0.619,3.316)$ & 0.4015 \\
\hline
\end{tabular}

Table 4. Cox model with hazard ratios and 95\% confidence intervals of COPD risk with medication treatment and covariates in patients with IBS compared to those without IBS. Crude HR = relative hazard ratio, $\mathrm{aHR}=$ Adjusted hazard ratio, $\mathrm{CI}=$ confidence interval, $\mathrm{IBS}=$ irritable bowel syndrome, $\mathrm{COPD}=$ chronic obstructive pulmonary disease ${ }^{\dagger}$ Model was adjusted for age, sex, and comorbidities.

\begin{tabular}{|l|l|l|l|l|l|}
\hline & Events & \multicolumn{1}{l|}{ Crude HR $(95 \%$ CI) } & \multicolumn{1}{l|}{ aHR $(95 \%$ CI) } \\
\hline
\end{tabular}

Table 5. Cox model with hazard ratios and $95 \%$ confidence intervals of COPD risk with frequency of outpatient visits for IBS. Crude $\mathrm{HR}=$ relative hazard ratio, $\mathrm{aHR}=$ Adjusted hazard ratio, $\mathrm{CI}=$ confidence interval, IBS $=$ irritable bowel syndrome, $\mathrm{COPD}=$ chronic obstructive pulmonary disease. ${ }^{\dagger}$ Model was adjusted for age, sex, and comorbidities.

value $=0.2123)$ (Table 2$)$. Those IBS patients with comorbidities including hypertension, coronary heart disease, ischemic stroke, hemorrhagic stroke, diabetes mellitus, renal insufficiency, depression, asthma, peptic ulcer, anxiety, sleep apnea, and osteoporosis exhibit a higher risk of developing COPD compared to the non-IBS group. However, only coronary heart disease, diabetes mellitus, depression, asthma, peptic ulcer, anxiety, sleep apnea, and osteoporosis comorbidities reached statistical significance (Table 2). This result indicates that there are many diseases associated with IBS patients, possible enhancing the risk of COPD presentation. The effects of IBS on COPD occurrence depending on age, gender, and the presence of comorbidities were demonstrated 


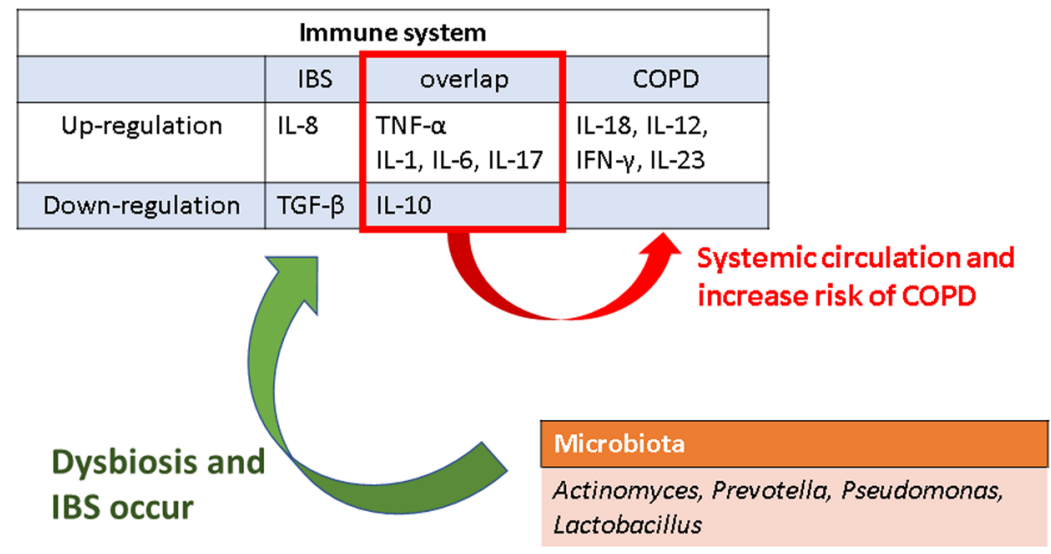

Figure 2. Possible mechanism and interaction between IBS and COPD.

in Table 3. Among age evaluation, the patients with IBS had higher risk of COPD than those without IBS in all group ( $\mathrm{aHR}=1.31-1.87, \mathrm{p}<0.001)$. The effect of IBS on COPD incidence was significantly higher in both men $(\mathrm{aHR}=1.50)$ and women $(\mathrm{aHR}=1.55)$ as well as participants both with $(\mathrm{aHR}=1.76)$ and without any comorbidities $(\mathrm{aHR}=1.39)$. Based on a stratification analysis of the $2,4,6,8$, and 10 -year follow-up periods, we found that within all of the follow-up period, the IBS cohort demonstrated a significantly increased risk of COPD compared to the non-IBS cohort, as shown in Fig. 1.

The hazard ratios of COPD associated with COPD medication and frequency of outpatient service in IBS patients. Recent COPD treatments are designed to slow disease progression. Thus, we further analyzed the association of hazard ratios with COPD regimens, including steroids, anti-cholinergics and beta-2 agonists between the IBS and non-IBS cohorts. Our results found that a higher incidence rate of COPD generally demonstrated significant differences between the IBS and non-IBS cohorts with steroids and anti-cholinergics taken for less than 28 days ( $p$ value $<0.0001$ ), but not for COPD medications taken for more than 28 days (Table 4). This indicates that patients with IBS had a higher possibility of developing COPD, and subsequently request COPD treatment. In addition, the regression model demonstrated that the aHR of COPD was higher in the IBS groups recording less than 18 times outpatient visits ( $\mathrm{aHR}=1.355-1.621, \mathrm{p}<0.05)$, reaching a statistical significance, rather than the IBS group recording more than 18 outpatient visits, $(\mathrm{aHR}=1.077,95 \% \mathrm{CI}=0.905-$ $1.281, \mathrm{p}=0.4046$ ) as compared to the non-IBS cohort (Table 5). This indicates that receiving treatment for IBS might decrease the incidence rate of COPD.

\section{Discussion}

Although previous studies have demonstrated that patients with COPD have an increased risk of IBS ${ }^{19,20}$, this is the first study using a population-based database to show that IBS potentially increases incidence rates of COPD. Our findings suggest that IBS patients had a significantly higher risk of developing COPD $(\mathrm{aHR}=1.512)$ after adjusting for sex, age, and major comorbidities.

Previous studies had mentioned the increased risk of inflammatory bowel disease (IBD) in COPD patients and the relationship of IBD and IBS. Labaraca et al. reported association between IBD and COPD and suggested potential mechanisms including increased pro-inflammatory cytokines, autoimmune antibodies, angiogenesis factors, and microbiome dysfunction, which leading to endothelial barrier dysfunction ${ }^{21}$. On the other hand, IBS and IBD shared many pathological mechanisms and overlapping symptoms. Spiller et al. demonstrated the shared mechanisms between IBS and IBD such as sustained submucosal inflammation, immune activation, dysbiosis in the gut and endothelial barrier dysfunction ${ }^{22}$. Other factors including stress, visceral hypersensitivity and the gut-brain axis were mentioned. Thus, we suppose that there would be some link between IBS and COPD. The immune system potentially plays a key role in the association between IBS and COPD. IBS patients have exhibited increased levels of TNF- $\alpha$, IL-1, IL-6, IL-8, IL-17, and decreased levels of TGF- $\beta$, IL-10 ${ }^{23-25}$. Furthermore, COPD results from sustained inflammation of the airways leading to the destruction of lung tissue and impairment of lung function. The inflammatory process is related to T cells, neutrophils, and macrophages triggered by cytokines. Recent studies have reported that COPD patients also had the effect to upregulate IL-1, IL-18, IL-6, IL-12, TNF- $\alpha$, IFN- $\gamma$, IL-23, IL-17 and downregulate IL-10 ${ }^{26-28}$. As such, there exist overlapping cytokine levels, including IL-10, TNF- $\alpha$, IL-1, IL-6, and IL-17 between IBS and COPD. Furthermore, studies have demonstrated the role of infection associated with disturbances of the microbiota in patients with IBS ${ }^{29}$. Such exposure to pathogens interferes with the gut barrier and increases gut permeability. However, inflammatory signals not only affect the gut but also circulate throughout the body causing visceral hyperalgesia associated with the brain-gut model of IBS pathophysiology ${ }^{30}$. The composition of lung microbiota in COPD patients has been shown to differ from that of healthy controls ${ }^{31,32}$. The immuno-stimulating properties of bacteria in the airway microbiota are very similar to gut microbiota ${ }^{14}$. While species like Actinomyces, Prevotella, Pseudomonas and Lactobacillus have been detected in both lungs and gastrointestinal tract ${ }^{14,31}$. Based on these findings, we suppose that dysregulation of 
the immune system, partially attributed to disturbed microbiota activity, could potentially explain the increased risk of COPD in patients with IBS. (Fig. 2).

Previous studies have pointed out that IBS affects females more than males ${ }^{33,34}$. In a meta-analysis study, the prevalence of IBS in women was higher in comparison with men $(14.0 \% \text { vs } 8.9 \% \text {; OR }=1.67)^{1}$. In the present study, we included IBS patients with 1:3 propensity score matching, with women comprising more than men $(\mathrm{M}: \mathrm{F}=45.3: 54.7)$, which is consistent with the IBS sex ratio previously reported. On the other hand, COPD affects men more than women, with a reported OR of 1.55 to $1.7^{35-38}$, a trend which is further confirmed in this study $(\mathrm{aHR}=1.610)$. We also note a steady increase of COPD diagnoses parallel with increasing age ${ }^{10}$. Pothirat et al. have reported that the general prevalence of COPD is higher in rural areas compared to urban areas $(6.8 \%$ vs $3.7 \%)^{39}$. This study found similar result that in Taiwan, the prevalence of COPD is the highest in the eastern, a rural areas compared to urban areas and reached a statistical significance to the northern area, a densely populated cities. Of note, the prevalence rate of comorbidities including COPD, coronary heart disease, diabetes mellitus, peptic ulcer, asthma, depression, anxiety, sleep apnea, and osteoporosis were significantly higher in patients with IBS than controls, confirming previous reports that IBS patients with the abovementioned comorbidities had a higher risk of developing COPD than those without comorbidities ${ }^{11,12}$.

Treatments for COPD include beta-2 agonists, anti-cholinergics, steroids (inhaled or oral), theophylline, and phosphodiesterase type 4 inhibitor inhibitors. In addition, IBS patients treated with beta- 2 agonists for less than 28 days had an increased incidence of developing COPD, with statistical significance. As reported, adrenergic stimulation triggers visceral hypersensitivity in IBS patients and rat model ${ }^{40-42}$; the mechanism of which is related to the polymorphism of adrenergic activation and elevation of transient receptor potential cation channel subfamily V member 1 (TRPV-1) sensitization ${ }^{42-44}$. Meanwhile, anticholinergic agents, which inhibit muscarinic receptors and cause smooth muscle relaxation ${ }^{45}$, may serve as effective antispasmodics and play an important role in control of abdominal pain in IBS patients ${ }^{46}$. Inhaled anticholinergic agents, such as ipratropium, oxitropium, and tiotropium also increase luminal diameter by smooth muscle relaxation and decreased submucosal gland mucin secretion in COPD patients ${ }^{47}$. We noted a decreased risk of COPD in IBS patients using anticholinergic agents for 28 to 84 days $(\mathrm{aHR}=0.181,95 \% \mathrm{CI}=0.020-1.677$, $\mathrm{p}$ value $=0.1325)$ and for more than 84 days $(\mathrm{aHR}=0.028,95 \% \mathrm{CI}=0.001-0.680, \mathrm{p}$ value $=0.0281)$ compared to the cohort receiving no drug treatment. The previously reported common physiological benefits of anticholinergic agents for treatment of both IBS and COPD are compatible with the findings of this study, although require further investigation ${ }^{45}$. We found that IBS patients with steroid treatment of more than 84 days had decreased incidence of COPD $(\mathrm{aHR}=0.122)$ with no significance. Steroids affect metabolism, blood pressure regulation, balance of mood, and anti-inflammation. Corticosteroids also coordinate with beta agonists by up-regulation of $\beta 2$-adrenoreceptors and modulate the immune system by regulation of $\mathrm{T}_{\text {cells }}{ }^{48}$, with a protective effect against development of COPD on patients using steroids for more than 84 days.

A higher rate of medical visits of IBS patients correspond with increased HR of COPD, indicating the severity and relapse rates of IBS patients; however, this consequence is decreased in patients recording medical visits of more than eighteen times. This suggests that the decreased incidence rate of COPD may be attributed to the active treatment of IBS. Importantly, IBS patients indeed exhibit an increased cumulative incidence rate of COPD, indicating significant association between these two diseases. However, the pathophysiology and specific interactions related to inflammation, infection, microbiota activity, and corresponding pathways between IBS and COPD remain unclear and require further investigation.

This is the first longitudinal population-based cohort study to evaluate the association between IBS and COPD. Due to the national health insurance (NHI) system covering $99.6 \%$ of the Taiwanese population as of 2017, this study has a large sample size yielding sufficient power for subgroup analysis, and enhanced potential to arrive at more conclusive results with adjustment for sex, age, and major comorbidities. NHIRD analysis is also inexpensive, with a very low loss of follow-up rate; thus, many studies using the available data are published annually. However, there remain several limitations and flaws to take into consideration. First, we used the ICD-9-CM algorithm to define diseases cited by clinical physicians. The precision of diagnoses was monitored by the Taiwan Bureau of National Health Insurance (an official insurance authority). The subjects were selected according to coding after a single inpatient admission, or three outpatient clinical visits to increase the validity and accuracy of the diagnoses. Second, the NHIRD does not offer information on potential confounding factors, including occupation, tobacco exposure, body mass index, physical activity, environmental/chemical exposure, or family history. We have analyzed the salary factor to discuss potential socioeconomic influence, which is consistent with other studies ${ }^{39}$. According to the Health Promotion Administration Report, tobacco usage was steady decrease in Taiwan recently. In 2017, 28.7\% of adults above the age of 18 years used tobacco products in Taiwan general population $^{49}$. However, there were no estimated data in IBS patients. Thus, we have adjusted the smoking factor for smoking-related diseases/conditions including personal history of tobacco use, tobacco use disorder, nonspecific abnormal results of pulmonary function studies, and other diseases of the lung (ICD 9: V15.82, 305.1, 794.2, 518) that are often diagnosed in the quit smoking clinic. Although underestimated smoking prevalence in our included patients, there was no statistical significance between two cohorts accordingly. Third, more detailed clinical evaluations, such as medical research council dyspnea scale, COPD assessment test, pulmonary function tests, serum laboratory data, or imaging results, are unavailable on the NHIRD. Thus, we cannot evaluate the severity of IBS in the subsequent risk of COPD, nor the COPD severity.

In conclusion, this retrospective cohort study indicates the significantly increased risk of developing COPD in patients with IBS. Instead of a causal relationship, both IBS and COPD may share a similar pathophysiological process associated with inflammation, infection, and microbiota interaction. However, further basic research is required to bridge these two diseases. Due to the increased COPD risk identified herein, it is critical for health care systems globally to enhance programs of early prevention and examination for COPD in patients with IBS. 


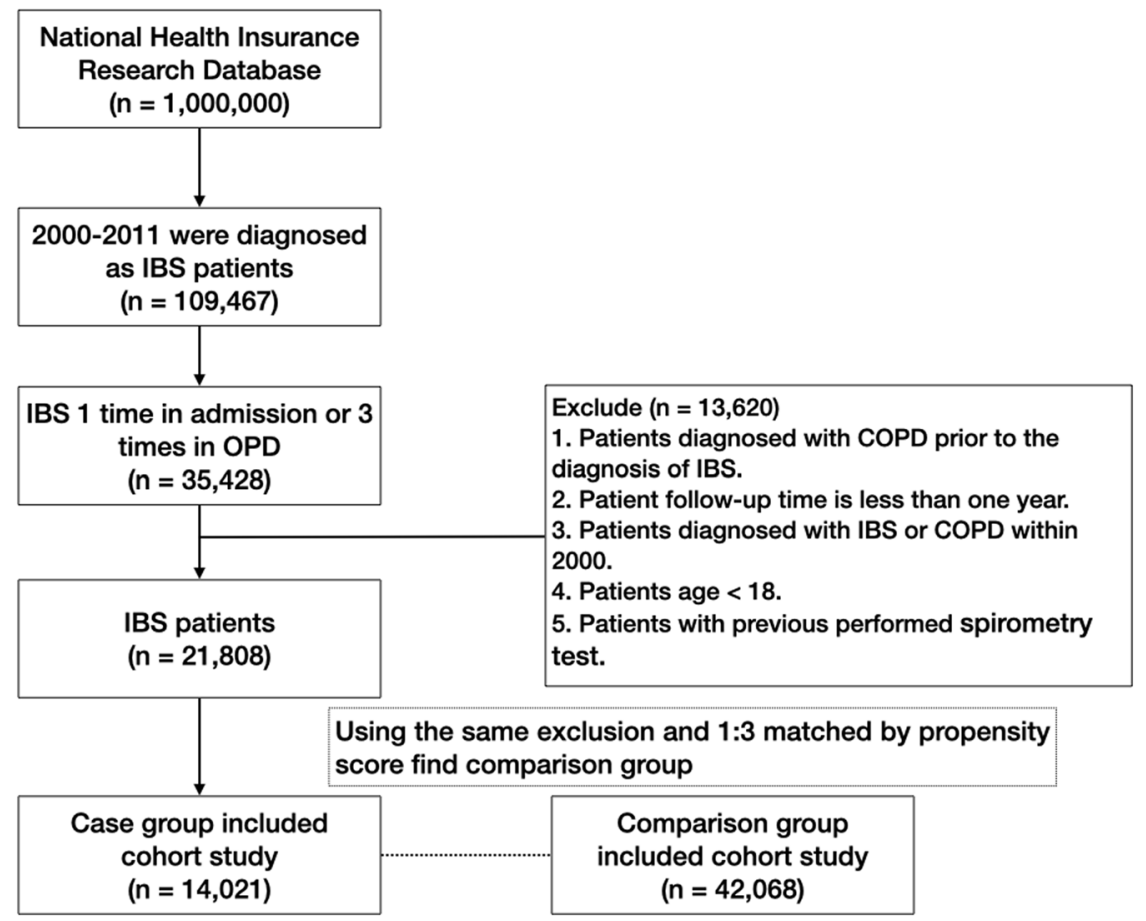

Figure 3. Flowchart of IBS (irritable bowel syndrome) and non-IBS enrollment from National Health Insurance Database (NHIRD) in Taiwan during 2000-2011.

\section{Materials and Methods}

Data sources. The data was retrieved from the (NHIRD), established by the National Health Research Institute (NHRI). The NHIRD is a population-based claims database covering over $99 \%$ of the Taiwanese population. The database is comprised of the claims data of over 23 million NHI enrollees during the years 2000 to 2011. All information, including age, sex, economic status, residence, diagnoses, dates of outpatient visits/admissions, and drug use have been verified. International Classification of Diseases, Ninth Revision, Clinical Modification (ICD-9-CM) codes is applied to classify diagnostic diseases.

Study population and variables. This is a retrospective cohort study conducted with the NHIRD. A flowchart illustrating subject selection for this study is shown in Fig. 3. Patients aged 18 years and older who were newly diagnosed with irritable bowel syndrome (IBS) (ICD-9-CM code 564.1), with follow-up of at least one year between January 1, 2000, and December 31, 2011, were enrolled and yielded 109,467 patients. Of these, we selected 35,428 patients with at least 3 OPD records or IBS diagnosis in admission. Only patients with a diagnosis of IBS were selected to ensure diagnostic validity. We excluded patients younger than 18 years of age, with follow-up of less than one year, with pre-existing COPD (ICD-9-CM code 491, 492, 496) before the diagnosis of IBS, with COPD within 1 year of IBS diagnosis, diagnosed with IBS or COPD within 2000, with previous performed spirometry test (code: 17003C, 17004B, 17006B, 17007B, 17019C) and those patients withdrawn from the NHIRD before diagnosis of IBS. These exclusion criteria yielded 13,620 patients for enrollment in the study. We then collected and analyzed demographic characteristics and claims data of both the IBS and non-IBS cohorts, matched by propensity score of 1:3. The case group and control group, consisting of 14,021 and 42,068 patients respectively, were included for further analysis. This study was approved by the Review Board and Ethics Committee of Taipei Medical University, Taiwan (TMU-JIRB No.: N201712044). All the datasets were decoded, so that the review board waived the requirement to sign informed consent from patients. All methods were carried out in accordance with relevant guidelines and regulations by ethics committee.

The demographic characteristics of the case and control cohorts including sex, age, residential area, salary, smoking-related diseases/conditions, common comorbidities, and drug intervention were taken into consideration as covariates to correct the analysis. The residential areas were divided into four main regions: northern Taiwan; central Taiwan; southern Taiwan; and eastern Taiwan. The study population was divided into six age groups: $18-29 ; 30-39 ; 40-49 ; 50-59 ; 60-69$; and $>69$ years old. The monthly salary income was categorized into 3 groups: $\leq$ NT $\$ 15,840$, NT\$15,841-25,000, $\geq$ NT $\$ 25,001$. Smoking status were defined as smoking-related diseases/conditions including personal history of tobacco use, tobacco use disorder, nonspecific abnormal results of pulmonary function studies, and other diseases of the lung (ICD 9: V15.82, 305.1, 794.2, 518) that are often diagnosed in the quit smoking clinic. Certain comorbid diseases were also recognized, including hypertension (ICD-9-CM code 401-405), coronary heart disease (ICD-9-CM code 410-414), ischemic stroke (ICD-9-CM code 433,434,436,437), hemorrhagic stroke (ICD-9-CM code 430-432), diabetes mellitus (ICD-9-CM code 250), renal insufficiency (ICD-9-CM code 585, 586, 588.8, 588.9), depression (ICD-9-CM code 296.2-296.36, 296.82, 300.4, 309.0, 309.1), asthma (ICD-9-CM code 493), Gastroesophageal reflux disease (GERD) (ICD-9-CM code 530.11, 530.81), peptic ulcer (ICD-9-CM code 531, 533), anxiety (ICD-9-CM code 293.84, 300.0, 300.00, 300.02, 300.09, 
309.21), sleep apnea (ICD-9-CM code 780.51, 780.53, 780.57), and osteoporosis (ICD-9-CM code 733-733.09). The common use of COPD medications including steroids, anti-cholinergics, and beta-2 agonists were also recruited to test categorical variables.

Statistical analysis. To reduce bias associated with confounding variables, we used propensity score-matched methods. To balance the measured covariate distribution in the cohorts, a propensity score for each patient was calculated by sex, age, area, urban, salary, smoking-related diseases/conditions, common comorbidities, and drug use. The propensity scores were calculated by using univariate and multivariate logistic regression to predict the probability of the occurrence of IBS. As such, the patients with IBS in line with 3 propensity score-matched subjects without IBS were comprised for further comparison.

The variables of the Cox regression model were used in this study. Categorical variables were demonstrated with counts and percentages, and those were further examined by Chi-square test to assess the association between the IBS and non-IBS cohorts. The mean age between the two groups was tested by two sample Student's t-test. Univariate and multivariable Cox regression was used to assess the risk of COPD and estimated hazard ratios (HRs) with confidence intervals (95\% CIs) between the two cohorts. In multivariate Cox regression, sex, age, salary, residential areas, smoking-related diseases/conditions, all comorbidities, and COPD medications were considered via adjustment. The Kaplan-Meier method to compare the cumulative incidence rate between IBS and non-IBS was applied for further management. The statistical analyses were performed using SAS software, version 9.4 (SAS Institute, Inc., Cary, NC, USA) and R software Version 3.4.3 (http://www.r-project.org), with level $\alpha=0.05$ to indicate statistical significance.

\section{Data availability}

The datasets used and/or analysed during the current study are available from the corresponding author on reasonable request.

Received: 14 December 2019; Accepted: 19 May 2020;

Published: $\mathrm{xx} \mathrm{xx}$ xxxx

\section{References}

1. Lovell, R. M. \& Ford, A. C. Global prevalence of and risk factors for irritable bowel syndrome: a meta-analysis. Clin Gastroenterol Hepatol 10, 712-721.e714, https://doi.org/10.1016/j.cgh.2012.02.029 (2012).

2. Bai, T. et al. Comparison of the Rome IV and Rome III criteria for IBS diagnosis: A cross-sectional survey. J Gastroenterol Hepatol 32, 1018-1025, https://doi.org/10.1111/jgh.13642 (2017).

3. Paudel, M. S. et al. Prevalence of Organic Colonic Lesions by Colonoscopy in Patients Fulfilling ROME IV Criteria of Irritable Bowel Syndrome. JNMA J Nepal Med Assoc 56, 487-492 (2018).

4. El-Salhy, M. Recent developments in the pathophysiology of irritable bowel syndrome. World J Gastroenterol 21, 7621-7636, https:// doi.org/10.3748/wjg.v21.i25.7621 (2015).

5. Sperber, A. D. \& Dekel, R. Irritable Bowel Syndrome and Co-morbid Gastrointestinal and Extra-gastrointestinal Functional Syndromes. J Neurogastroenterol Motil 16, 113-119, https://doi.org/10.5056/jnm.2010.16.2.113 (2010).

6. Hausteiner-Wiehle, C. \& Henningsen, P. Irritable bowel syndrome: relations with functional, mental, and somatoform disorders. World J Gastroenterol 20, 6024-6030, https://doi.org/10.3748/wjg.v20.i20.6024 (2014).

7. Fond, G. et al. Anxiety and depression comorbidities in irritable bowel syndrome (IBS): a systematic review and meta-analysis. Eur Arch Psychiatry Clin Neurosci 264, 651-660, https://doi.org/10.1007/s00406-014-0502-z (2014).

8. Shen, T. C. et al. Bidirectional Association between Asthma and Irritable Bowel Syndrome: Two Population-Based Retrospective Cohort Studies. PLoS One 11, e0153911, https://doi.org/10.1371/journal.pone.0153911 (2016).

9. Marcoa, R. et al. Classification of Chronic Obstructive Pulmonary Disease (COPD) according to the new Global Initiative for Chrnnic Ohstructive I uno Disease (GOIJD) 2017: Comparison with GOLD 2011. Copd 15, 21-26 (2018).

Published online: 19 June $2020 \quad$ nt COPD International Patient Survey: methods, COPD prevalence, and disease burden in 2012-2013. Int J Chron Obstruct Pulmon Dis 9, 597-611, https://doi.org/10.2147/copd.s61854 (2014).

11. Hillas, G., Perlikos, F., Tsiligianni, I. \& Tzanakis, N. Managing comorbidities in COPD. Int J Chron Obstruct Pulmon Dis 10, 95-109, https://doi.org/10.2147/copd.s54473 (2015).

12. Putcha, N., Drummond, M. B., Wise, R. A. \& Hansel, N. N. Comorbidities and Chronic Obstructive Pulmonary Disease: Prevalence, Influence on Outcomes, and Management. Semin Respir Crit Care Med 36, 575-591, https://doi.org/10.1055/s-0035-1556063 (2015).

13. Decramer, M., Janssens, W. \& Miravitlles, M. Chronic obstructive pulmonary disease. Lancet 379, 1341-1351, https://doi. org/10.1016/s0140-6736(11)60968-9 (2012).

14. Malhotra, R. \& Olsson, H. Immunology, genetics and microbiota in the COPD pathophysiology: potential scope for patient stratification. Expert Rev. Respir Med 9, 153-159, https://doi.org/10.1586/17476348.2015.1000865 (2015).

15. Yazar, A. et al. Respiratory symptoms and pulmonary functional changes in patients with irritable bowel syndrome. Am J Gastroenterol 96, 1511-1516, https://doi.org/10.1111/j.1572-0241.2001.03748.x (2001).

16. Kim, J. et al. Association between chronic obstructive pulmonary disease and gastroesophageal reflux disease: a national crosssectional cohort study. BMC Pulm Med 13, 51, https://doi.org/10.1186/1471-2466-13-51 (2013).

17. Kim, S. W., Lee, J. H., Sim, Y. S., Ryu, Y. J. \& Chang, J. H. Prevalence and risk factors for reflux esophagitis in patients with chronic obstructive pulmonary disease. Korean J Intern Med 29, 466-473, https://doi.org/10.3904/kjim.2014.29.4.466 (2014).

18. Niklasson, A., Strid, H., Simren, M., Engstrom, C. P. \& Bjornsson, E. Prevalence of gastrointestinal symptoms in patients with chronic obstructive pulmonary disease. Eur J Gastroenterol Hepatol 20, 335-341, https://doi.org/10.1097/MEG.0b013e3282f2d0ec (2008).

19. Chetty, U. et al. Chronic obstructive pulmonary disease and comorbidities: a large cross-sectional study in primary care. $\mathrm{Br} J \mathrm{Gen}$ Pract 67, e321-e328, https://doi.org/10.3399/bjgp17X690605 (2017).

20. Hanlon, P. et al. Examining patterns of multimorbidity, polypharmacy and risk of adverse drug reactions in chronic obstructive pulmonary disease: a cross-sectional UK Biobank study. BMJ Open 8, e018404, https://doi.org/10.1136/bmjopen-2017-018404 (2018).

21. Spiller, R. \& Major, G. IBS and IBD - separate entities or on a spectrum? Nat Rev Gastroenterol Hepatol 13, 613-621, https://doi. org/10.1038/nrgastro.2016.141 (2016).

22. Labarca, G. et al. Association between inflammatory bowel disease and chronic obstructive pulmonary disease: a systematic review and meta-analysis. BMC Pulm Med 19, 186, https://doi.org/10.1186/s12890-019-0963-y (2019). 
23. Rana, S. V. et al. Pro-inflammatory and anti-inflammatory cytokine response in diarrhoea-predominant irritable bowel syndrome patients. Trop Gastroenterol 33, 251-256 (2012).

24. Buckley, M. M., O’Mahony, S. M. \& O'Malley, D. Convergence of neuro-endocrine-immune pathways in the pathophysiology of irritable bowel syndrome. World J Gastroenterol 20, 8846-8858, https://doi.org/10.3748/wjg.v20.i27.8846 (2014).

25. Bashashati, M. et al. Cytokine imbalance in irritable bowel syndrome: a systematic review and meta-analysis. Neurogastroenterol Motil 26, 1036-1048, https://doi.org/10.1111/nmo.12358 (2014)

26. Atamas, S. P., Chapoval, S. P. \& Keegan, A. D. Cytokines in chronic respiratory diseases. F1000 Biol Rep 5, 3, https://doi.org/10.3410/ b5-3 (2013).

27. Huang, G. et al. Neutrophilic Inflammation in the Immune Responses of Chronic Obstructive Pulmonary Disease: Lessons from Animal Models. J Immunol Res 2017, 7915975, https://doi.org/10.1155/2017/7915975 (2017).

28. Yadava, K. et al. Microbiota Promotes Chronic Pulmonary Inflammation by Enhancing IL-17A and Autoantibodies. Am J Respir Crit Care Med 193, 975-987, https://doi.org/10.1164/rccm.201504-0779OC (2016).

29. Ringel, Y. \& Ringel-Kulka, T. The Intestinal Microbiota and Irritable Bowel Syndrome. J Clin Gastroenterol 49(Suppl 1), S56-59, https://doi.org/10.1097/mcg.0000000000000418 (2015).

30. Holtmann, G. J., Ford, A. C. \& Talley, N. J. Pathophysiology of irritable bowel syndrome. Lancet Gastroenterol Hepatol 1, 133-146, https://doi.org/10.1016/s2468-1253(16)30023-1 (2016).

31. Sze, M. A. et al. The lung tissue microbiome in chronic obstructive pulmonary disease. Am J Respir Crit Care Med 185, 1073-1080, https://doi.org/10.1164/rccm.201111-2075OC (2012).

32. Sze, M. A., Hogg, J. C. \& Sin, D. D. Bacterial microbiome of lungs in COPD. Int JChron Obstruct Pulmon Dis 9, 229-238, https://doi. org/10.2147/copd.S38932 (2014).

33. Lovell, R. M. \& Ford, A. C. Effect of gender on prevalence of irritable bowel syndrome in the community: systematic review and meta-analysis. Am J Gastroenterol 107, 991-1000, https://doi.org/10.1038/ajg.2012.131 (2012).

34. Naeem, S. S. et al. Prevalence and factors associated with irritable bowel syndrome among medical students of Karachi, Pakistan: a cross-sectional study. BMC Res Notes 5, 255, https://doi.org/10.1186/1756-0500-5-255 (2012).

35. Han, M. K. et al. Gender and chronic obstructive pulmonary disease: why it matters. Am J Respir Crit Care Med 176, 1179-1184, https://doi.org/10.1164/rccm.200704-553CC (2007).

36. Aryal, S., Diaz-Guzman, E. \& Mannino, D. M. Influence of sex on chronic obstructive pulmonary disease risk and treatment outcomes. Int J Chron Obstruct Pulmon Dis 9, 1145-1154, https://doi.org/10.2147/copd.S54476 (2014).

37. Lam, H. T. et al. Prevalence of COPD by disease severity in men and women in northern Vietnam. Copd 11, 575-581, https://doi.or $\mathrm{g} / 10.3109 / 15412555.2014 .898039$ (2014).

38. Waatevik, M. et al. Increased prevalence of chronic obstructive pulmonary disease in a general population. Respir Med 107, 1037-1045, https://doi.org/10.1016/j.rmed.2013.04.008 (2013).

39. Pothirat, C. et al. A comparative study of COPD burden between urban vs rural communities in northern Thailand. Int J Chron Obstruct Pulmon Dis 10, 1035-1042, https://doi.org/10.2147/copd.S82303 (2015).

40. Abrahamsson, H. \& Dotevall, G. Effects of propranolol on colonic pressure in patients with irritable bowel syndrome. Scand J Gastroenterol 16, 1021-1024 (1981).

41. Park, J. H. et al. Increased beta-adrenergic sensitivity correlates with visceral hypersensitivity in patients with constipationpredominant irritable bowel syndrome. Dig Dis Sci 50, 1454-1460 (2005)

42. Zhu, L. et al. Adrenergic stimulation sensitizes TRPV1 through upregulation of cystathionine beta-synthetase in a rat model of visceral hypersensitivity. Sci Rep 5, 16109, https://doi.org/10.1038/srep16109 (2015).

43. Sikander, A. et al. Association of alpha $2 \mathrm{~A}$ adrenergic receptor gene (ADRAlpha2A) polymorphism with irritable bowel syndrome, microscopic and ulcerative colitis. Clin Chim Acta 411, 59-63, https://doi.org/10.1016/j.cca.2009.10.003 (2010).

44. Zhang, C. et al. Adrenergic beta2-receptors mediates visceral hypersensitivity induced by heterotypic intermittent stress in rats. PLoS One 9, e94726, https://doi.org/10.1371/journal.pone.0094726 (2014).

45. Peretto, I., Petrillo, P. \& Imbimbo, B. P. Medicinal chemistry and therapeutic potential of muscarinic M3 antagonists. Med Res Rev 29, 867-902, https://doi.org/10.1002/med.20158 (2009).

46. Ruepert, L. et al. Bulking agents, antispasmodics and antidepressants for the treatment of irritable bowel syndrome. Cochrane Database Syst Rev, Cd003460, https://doi.org/10.1002/14651858.CD003460.pub3 (2011).

47. Kim, V. \& Criner, G. J. Chronic bronchitis and chronic obstructive pulmonary disease. Am J Respir Crit Care Med 187, 228-237, https://doi.org/10.1164/rccm.201210-1843CI (2013).

48. Yang, L. et al. Relationship between the anti-inflammatory properties of salmeterol/fluticasone and the expression of CD4(+) CD25(+)Foxp3(+) regulatory T cells in COPD. Respir Res 12, 142, https://doi.org/10.1186/1465-9921-12-142 (2011)

49. Health Promotion Administration. Taiwan Tobacco Control Annual Report 2018. Health Promotion Administration, Taiwan (2018).

\section{Acknowledgements}

This work was supported and funded by the Ministry of Science and Technology of Taiwan (MOST 108-2320B-039-022), Health and Welfare Surcharge of Tobacco Products, China Medical University Hospital Cancer Research Center of Excellence (MOHW 108-TDU-B-212-124024), China Medical University Hospital (DMR108-007, DMR-108-009, DMR-108-044 and CRS-108-001), An-Nan Hospital, China Medical University (ANHRF-108-06 and ANHRF-108-08) and the Chinese Medicine Research Center, China Medical University, under the Higher Education Sprout Project, Ministry of Education (CMRC-CHM-1) in Taiwan. The authors would like to thank James Waddell for the critical reading and revision of our manuscript.

\section{Author contributions}

H.-C.L. wrote the manuscript. H.-J.L. and J.-W.C. interpreted the data and administrative support. Y.-W.K. and K.-H.W. collected, assembled, and analyzed the data. B.-C.S. provided the data and amended the manuscript. S.-T.H. designed, conceived the study and amended the manuscript. All of the authors approved the final manuscript.

\section{Competing interests}

The authors declare no competing interests.

\section{Additional information}

Correspondence and requests for materials should be addressed to B.-C.S. or S.-T.H.

Reprints and permissions information is available at www.nature.com/reprints. 
Publisher's note Springer Nature remains neutral with regard to jurisdictional claims in published maps and institutional affiliations.

(c) (i) Open Access This article is licensed under a Creative Commons Attribution 4.0 International License, which permits use, sharing, adaptation, distribution and reproduction in any medium or format, as long as you give appropriate credit to the original author(s) and the source, provide a link to the Creative Commons license, and indicate if changes were made. The images or other third party material in this article are included in the article's Creative Commons license, unless indicated otherwise in a credit line to the material. If material is not included in the article's Creative Commons license and your intended use is not permitted by statutory regulation or exceeds the permitted use, you will need to obtain permission directly from the copyright holder. To view a copy of this license, visit http://creativecommons.org/licenses/by/4.0/.

(c) The Author(s) 2020 